\title{
Chitosan-graft-poly(n-butyl acrylate) copolymer: Synthesis and characterization of a natural/synthetic hybrid material
}

\author{
Pablo Anbinder ${ }^{\mathrm{b}}$, Carlos Macchi ${ }^{\mathrm{b}}$, Javier Amalvy ${ }^{\mathrm{a}, \mathrm{c}, \mathrm{d}, *}$, Alberto Somoza $^{\mathrm{b}}$ \\ a Instituto de Investigaciones Físico-Químicas Teóricas y Aplicadas (INIFTA)-(CONICET CCT La Plata-UNLP), Diag. 113 y 64, 1900 La Plata, Argentina \\ ${ }^{\mathrm{b}}$ Instituto de Fisica de Materiales Tandil (IFIMAT-UNCPBA) and CIFICEN (UNCPBA-CONICET-CICPBA), Pinto 399 B7000GHG, Tandil, Argentina \\ ' Cátedra de Materiales Poliméricos-Facultad de Ingeniería (UNLP), Calle 1 y 47, 1900 La Plata, Argentina \\ a Centro de Investigación y Desarrollo en Ciencia y Tecnología de los Materiales (CITEMA)-Facultad Regional La Plata (Universidad Tecnológica Nacional), \\ Av. 60 y 124, 1900 La Plata, Argentina
}

Keywords:
Biopolymer
Chitosan
n-Butyl acrylate
Graft
Thermal and structural properties

\begin{abstract}
A B S T R A C T
Two chitosan polymers with different deacetylation degree and molecular weight were subjected to grafting reactions with the aim to enhance the properties of these bio-based materials. Specifically, $n$-butyl acrylate in different proportions was grafted onto two different deacetylation degree (DD\%) chitosan using radical initiation in a surfactant free emulsion system. Infrared spectroscopy was used to confirm grafting and products grafting percentage and efficiency were evaluated against acrylate/chitosan ratio and DD\%. Thermal and structural properties and the behavior against water of the raw and grafted biopolymers were studied using several experimental techniques: differential scanning calorimetry, transmission electron microscopy, dynamic light scattering, water swelling, contact angle and positron annihilation lifetime spectroscopy. The influence of the grafting process on the morphological and physicochemical properties of the prepared natural/synthetic hybrid materials is discussed.
\end{abstract}

\section{Introduction}

Nowadays, an important percentage of materials used in the different packaging industries is made from fossil fuels and are undegradable. For this reason, packaging materials for short-term storage, like foodstuff, represent a serious global environmental problem (Kirwan \& Strawbridge, 2003). Until recently, polymeric materials were designed in order to fulfill the specific function for which they were required (i.e., packages to adequately protect the contents). Currently, in addition to satisfying its function, polymeric materials are al so designed to minimize environmental impact. The term "degradable" cover all those polymers that can be degraded by the action of different media or agents such as light (photo-), moisture or water (hydro-), oxygen-heat-light (oxo-) microorganisms (bio-) or the presence of certain additives or catalysts that favor or accelerate the decomposition and degradation. In particular, biodegradable polymers are those that are going to be degraded by enzymatic action of certain bacteria or microorganisms which

\footnotetext{
* Corresponding author.

E-mail addresses: psanbinder@inifta.unlp.edu.ar

(P. Anbinder), cmacchi@exa.unicen.edu.ar (C. Macchi), jamalvy@inifta.unlp.edu.ar, jamalvy@gmail.com (J. Amalvy), asomoza@exa.unicen.edu.ar (A. Somoza).
}

will consume and fully absorb the polymer until convert it into energy, methane, water and carbon dioxide. As regards, the term biopolymer is devoted to those that are made from renewable sources and/or are biodegradable. First, a biopolymer not necessarily must be biodegradable, as new polyethylenes based on renewable sources (e.g., sugar cane); and, secondly, a biodegradable polymer does not necessarily have to come from renewable sources, as is the case of some polyesters derived from petroleum. Within biopolymers, there are bio-based polymers $100 \%$ made on renewable sources, and also those that are made in part from renewable sources and partially from non-renewable sources. At this time, there is no agreement or a clear definition about what would be the minimum percentage of renewable raw material that should have a polymer to be classified as a biopolymer. Those partially bio-based materials, although they only have a certain percentage of renewable raw materials, could also provide an important impact on the environmental issues.

Biopolymers produced from renewable resources or from different industry discards are an innovating answer to replace conventional petroleum-based products. However, to obtain useful and competitive products some of the properties of this kind of materials (relatively poor mechanical properties, brittleness at room temperature, moisture sensitivity, etc.) should be enhanced. The potential of the bio-based products have been widely men- 
tioned, but the replacement of the non-degradable plastics, is still an utopia. The common approach to tune their behaviors consists on the elaboration of multiphase materials, e.g., blends (Averous, Moro, Dole, \& Fringant, 2000; van den Broek, Knoop, Kappen, \& Boeriu, 2015), composites (Chivrac, Pollet, \& Avérous, 2009; Xie, Pollet, Halley, \& Avérous, 2013) or hybrid materials (CostaJúnior, Barbosa-Stancioli, Mansur, Vasconcelos, \& Mansur, 2009; Vartiainen, Tammelin, Pere, Tapper, \& Harlin, 2010).

Chitosan is the partially deacetylated form of the chitin, a natural polysaccharide extracted from the crab, shrimps and other crustaceans' skeleton. In general, chitosan occurs as $\mathrm{N}$-acetylglucosamine and $\mathrm{N}$-glucosamine copolymer that are randomly or block distributed throughout the biopolymer chain (Rinaudo, 2006). This distribution (random or block wise) may influence the solubility of the polymer and then the interchain interactions due to the $\mathrm{H}$-bonds and the hydrophobic character of the acetyl groups (Rinaudo, 2012). Chitosan has been listed as a "promising" or "potential" biomaterial for over forty years, due to its properties and the diverse biological activities reported (Muzzarelli, Isolati, \& Ferrero, 1974; Masri \& Friedman, 1972), but chitosan-based products were slow to enter into the market due to poor reproducibility in the production processes and product performances. However, chitosan products based on the structural properties of the chitosans, but also on their biological functionalities, are increasingly appearing on the markets. Recently, demands for chitosan have for the first time exceeded the supply of high quality materials available. In 2014, chitosan has been classified as a "basic substance" by the European Commission, offering great trade opportunities.

Chitosan-based biopolymers have great potential as packaging material, due to its inherent antimicrobial activity, non-toxicity and biodegradability (Harish Prashanth \& Tharanathan, 2003; Rhim \& $\mathrm{Ng}, 2007)$. However, as mentioned for other biopolymers, chitosan has relatively poor mechanical properties, mainly involving low flexibility and brittleness, and reduced performance against water.

Chemical modification of chitosan, specially grafting with different polymers is an important topic in the production of bio-based materials with enhanced properties. In this way, chitosan presents two types of reactive sites which can (potentially) be modified by grafting: the C-2 free amino groups on deacetylated units and the C-3 and C-6 hydroxyl groups in either acetylated or deacetylated units (Lloyd, Kennedy, Methacanon, Paterson, \& Knill, 1998).

Acrylic monomers were used to modify chitin (Ifuku, Iwasaki, Morimoto, \& Saimoto, 2011) and different chitosan derivatives, such as phthaloylchitosan (Li, Liu, Shen, \& Fang., 2005), thiolated chitosan (Bravo-Osuna, Schmitz, Bernkop-SchnÛrch, Vauthier, \& Ponchel, 2006) or carboxymethyl chitosan (Chen \& Tan, 2006). Regarding chitosan grafted systems, many of vinyl-derivatives monomers such as methylmethacrylate (MMA) (Harish Prashanth \& Tharanathan, 2003; Singh, Sharma, Tripathi, \& Sanghi, 2009), acrylonitrile (AN) (Harish Prashanth \& Tharanathan, 2003; Shankar, Gomathi, Vijayalakshmi, \& Sudha, 2014), acrylamide (Özbaș \& Gürdağ, 2015), 2-hydroxyethyl methacrylate (HEMA) (Singh \& Ray, 1998; Ávila, Bierbrauer, Pucci, López-González \& Strumia, 2012), acrylic acid (AA) (Ávila et al., 2012; Ge \& Wang, 2014) and glycidylmethacrylate (Chellapandian \& Krishnan, 1998), among others, were used to copolymerize this biopolymer.

For this work, n-butyl acrylate was chosen because of its homopolymer presents low glass transition temperature $\left(T_{\mathrm{g}}\right)$ and hydrophobic characteristics, two important properties that could be tailored to chitosan to get the desired results in the final product, i.e. improve its water resistance and mechanical properties.

Graft copolymerization of vinyl monomers onto chitosan and other natural polymers using free radicals initiation is a very fascinating research field with many future industrial possibilities. This technique enables the production of a wide range of new poly-
Table 1

Sample identification.

\begin{tabular}{lll}
\hline Sample & Chitosan polymer & Weight ratio Q:BuA \\
\hline QS & Sigma (78.2 DD\%) & $1: 0$ \\
QP & Parafarm (95.2 DD\%) & $1: 0$ \\
QSA50 & Sigma & $1: 0.5$ \\
QSA100 & & $1: 1$ \\
QPA50 & Parafarm & $1: 0.5$ \\
QPA100 & & $1: 1$ \\
\hline
\end{tabular}

mer materials with desired properties that are based on chemical combinations of natural and synthetic polymers.

Briefly, in the present work two chitosan polymers with different deacetylation degree and molecular weight were subjected to grafting reactions with $n$-butyl acrylate in different proportions. Micro and nanostructure and thermal properties of raw and grafted samples were studied and compared, as well as the be havior against water.

\section{Methods}

\subsection{Materials}

2.1.1. The materials and chemicals used in this work were

Chitosan: medium molecular weight chitosan (QS) with a 78.2\% deacetylation degree and $M_{\mathrm{V}} 450,000$ (Sigma); Chitosan (QP) with a 95.2\% deacetylation degree and $M_{\mathrm{V}}$ 310,000 (Parafarm); $n$ butylacrylate (BuA, Sigma); acetic acid solution $0.165 \mathrm{M}(1 \% \mathrm{v} / \mathrm{v})$; acetone (Merck); sodium hydroxide $(\mathrm{NaOH}$, Anedra); potassium persulphate (KPS, Anedra). All reagents were of analytical grade and used without purification, except BuA which was purified according to the method reported by Armarego and Chai, (2013, Chap. 4)). Chitosans $M_{\mathrm{V}}$ was determined by capilar viscosimetry and DD \% was obtained by FT-IR (Baxter, Dillon, Taylor, \& Roberts, 1992) and confirmed by potenciometry (Jiang, Chen, \& Zhong, 2003).

\subsection{Chitosan-graft-butyl acrylate synthesis}

Chitosan solution ( $2 \% \mathrm{w} / \mathrm{v}$ in $1 \% \mathrm{v} / \mathrm{v}$ acetic acid) was placed in a flat bottomed three necked flask. A nitrogen purge was made throughout one hour at room temperature (RT, i.e., $25^{\circ} \mathrm{C}$ ) through the stirred solution. Then, the temperature was set at $60^{\circ} \mathrm{C}$ and a freshly prepared KPS solution $(0.741 \mathrm{mM}$ in the minimum quantity of water, around $1 \mathrm{~mL}$ ) was added. Five minutes later, dropwise addition (78-156 mM) of $n$-butyl acrylate was made during $20 \mathrm{~min}$.

The reaction was conducted at $60^{\circ} \mathrm{C}$ for $24 \mathrm{~h}$ with stirring and $\mathrm{N}_{2}$ purge. Finally, another $0.370 \mathrm{mM} \mathrm{KPS}$ was added to ensure the complete polymerization of the acrylic component. Table 1 resumes the dispersions synthesized and their nomenclature.

\subsection{Film preparation}

$12 \mathrm{~mL}$ of the solution were cast on a $10 \mathrm{~cm}$ diameter glass Petri dishes, dried at RT for $48 \mathrm{~h}$ and then another $4 \mathrm{~h}$ at $60^{\circ} \mathrm{C}$. After cooling, films were neutralized with $1 \mathrm{~N} \mathrm{NaOH}$ to allow the peeling off, washed with distilled water and dry at RT in a PTFE plate.

\subsection{Grafting percentage and reaction efficiency}

To eliminate the acrylate homopolymer copolymer films were extracted by Soxhlet with acetone for $8 \mathrm{~h}$. The gain in weight of the copolymer was measured as grafting percentage (Eq. (1)), where $W_{\mathrm{g}}$ is the weight in grams of the grafted chitosan and $W_{0}$ is the weight of native chitosan. On the other hand, reaction efficiency 
was calculated through Eq. (2), where Q/A is the chitosan/n-butyl acrylate weight ratio.

$$
\begin{aligned}
& G \%=\left(\frac{W_{\mathrm{g}}-W_{0}}{W_{0}}\right) \times 100 \\
& \mathrm{Eff} \%=\left(\frac{G \%}{Q / A}\right) \times 100
\end{aligned}
$$

\subsection{Infrared spectroscopy (FT-IR)}

FT-IR spectra of films were acquired using a FT-IR Nicolet 380 spectrometer with a Ge monoreflection ATR accessory, taking 64 scans per spectra, with a resolution of $4 \mathrm{~cm}^{-1}$. The analyzed surface was that exposed to air in the casting step.

\subsection{X-ray diffractometry $(X R D)$}

Powder X-ray diffraction patterns of chitosan and grafted samples were obtained by using a Philips PW 3710 powder diffractometer operating under the following conditions: $20 \mathrm{kV}$ and $20 \mathrm{~mA}$ with $\mathrm{CuK} \alpha$ radiation at $\lambda=1.54184 \AA$. The relative intensity was recorded in the scattering range $(2 \theta)$ of $5-40^{\circ}$. To obtain the powders, previously the film samples were immersed in liquid nitrogen and milled.

\subsection{Transmission electron microscopy (TEM)}

TEM images were obtained using a JEM 1200 EX II-JEOL microscope. Diluted dispersions were dried onto carbon-coated copper grids without staining.

\subsection{Particle size}

Particle size was determined by means of dynamic light scattering on a Zetasizer Nano (Nano ZSizer-ZEN3600, Malvern, U.K.). Dispersions were diluted with milli-Q water and measured at RT. Besides the Z-average diameter, the polydispersity index (PdI) was obtained using Eq. (3) as follows:

$\mathrm{PdI}=\frac{D_{\mathrm{v}}}{D_{\mathrm{n}}}$

where $D_{v}$ and $D_{n}$ are the volume and number average particle diameters, respectively.

\subsection{Differential scanning calorimetry (DSC)}

DSC studies were performed in a TA-Q20 calorimeter. Thermograms were acquired at $10^{\circ} \mathrm{C} / \mathrm{min}$ from $-80^{\circ} \mathrm{C}$ to $30^{\circ} \mathrm{C}$, with $\mathrm{Ar}$ purge $(50 \mathrm{~mL} / \mathrm{min})$.

\subsection{Degree of swelling}

The water swelling degree was evaluated by an immersion assay at RT, using circular films of $25 \mathrm{~mm}$ diameter. Dry samples $\left(W_{0}\right)$ were immersed in distilled water, removed daily from the solution, quickly wiped with a filter paper and weighted until reach a constant weight $\left(W_{\mathrm{s}}\right)$. The equilibrium water uptake (WS\%) of the films was determined according to the following expression:

$\mathrm{WS} \%=\left(\frac{W_{\mathrm{S}}-W_{0}}{W_{0}}\right) \times 100$

\subsection{Contact angle}

Contact angle measurements were carried out using a Model 500 goniometer (Ramé-Hart Instrument Co., USA) in the contact angle mode. Images were analyzed using the DROP image Advanced v2.2 software. All the tests were performed on the airfacing surfaces of the samples. To calculate the mean static contact angle $\theta$, six measurements on different points were performed.

\subsection{Positron annihilation lifetime spectroscopy (PALS)}

The positron lifetime spectrometer used consisted on a "fast-fast" coincidence system with a resolution of 340 ps. PALS spectra were recorded at RT with a total number of $1.5 \times 10^{6}$ coincidence counts using a $0.2 \mathrm{MBq}$ sealed source of ${ }^{22} \mathrm{NaCl}$ deposited onto a thin Kapton foil $\left(1.08 \mathrm{mg} / \mathrm{cm}^{2}\right)$. The source was sandwiched between two "identical pieces" of the sample material. These "pieces" were prepared following the same procedure used by Consolati, Levi, and Turri (2001); i.e. stacking several layers of the film in order to stop all the injected positrons. In each case, pieces were compressed in order to eliminate the air gaps between two subsequent layers.

According to the common interpretation for PALS measurements in polymers, spectra were deconvoluted into three discrete lifetime components using the LT10 software (Giebel \& Kansy, 2011).

In many materials, when the open volume reaches the subnanometer size, positronium (Ps) can be formed. Positronium is formed in two states: a singlet state, para-positronium (p-Ps) and a triplet state, ortho-positronium (o-Ps). In vacuum, p-Ps annihilates into two gamma rays of $511 \mathrm{keV}$ with a lifetime of $125 \mathrm{ps}$, whereas o-Ps annihilates into three gamma rays with a lifetime of $142 \mathrm{~ns}$ The lifetime of o-Ps formed in open volumes is reduced by the $2 \gamma$ pick-off process in which positron annihilates with an electron of opposite spin belonging to molecules of the walls of the cavity. The size of the nano or sub-nanoholes is directly linked to the reduced lifetime of the o-Ps.

As usual, we ascribed the longest lifetime component $\tau_{3}$ to ortho-Ps decay in the nanoholes forming the free volume; i.e. $\tau_{3}=\tau_{0}$-Ps. The intermediate component $\tau_{2}(0.35-0.50 \mathrm{~ns})$ is attributed to positrons annihilated in low electron density regions of the structure. The shortest component $\tau_{1}(0.1-0.2 \mathrm{~ns})$ is due to positrons annihilated into the bulk and to p-Ps annihilations. A correlation is possible between the longest lifetime component and the size of the hole (in spherical approximation of nanoholes of radii $R$ ), as expressed using a simple quantum mechanical model (Tao, 1972; Eldrup, Lightbody, \& Sherwook, 1981):

$\tau_{o-\mathrm{Ps}}=0.5\left[\frac{\Delta R}{R+\Delta R}+\frac{1}{2 \pi} \sin \left(\frac{2 \pi R}{R+\Delta R}\right)\right]^{-1}$

where $\tau_{\mathrm{o}-\mathrm{Ps}}$ is given in $\mathrm{ns}, R$ in $\mathrm{nm}$ and $\Delta R=0.166 \mathrm{~nm}$ (Nakanishi, Wang, \& Jean, 1998).

In the present work, we have used the simplest approach to get the fractional free volume $f_{\mathrm{v}}$ from the positron data, where the number of the nanoholes forming the free volume in polymers is represented by the intensity associated with the o-Ps lifetime (Kobayashi et al., 1989; Wang, Nakanishi, Jean, \& Sandreczky, 1990). Under this frame, $f_{\mathrm{v}}$ can be assumed to be proportional to the number of nanoholes and to the average volume of each nanohole. Then, the following semi-empirical equation can be used:

$f_{\mathrm{V}}=A \times v_{\mathrm{h}} \times I_{0-\mathrm{Ps}}$

where $v_{\mathrm{h}}$ (in $\AA^{3}$ ) is the volume of the nanohole calculated using the spherical radius $R$ obtained using Eq. (5), the intensity $I_{0-P s}$ is given in percentage and $A=0.0018$ is an empirical scaling constant (Jean, 1990).

\section{Results and discussion}

Stable dispersions with $\approx 3 \% \mathrm{w} / \mathrm{v}$ of solids were obtained by the synthesis methods used in this work, with no final monomer odor. 

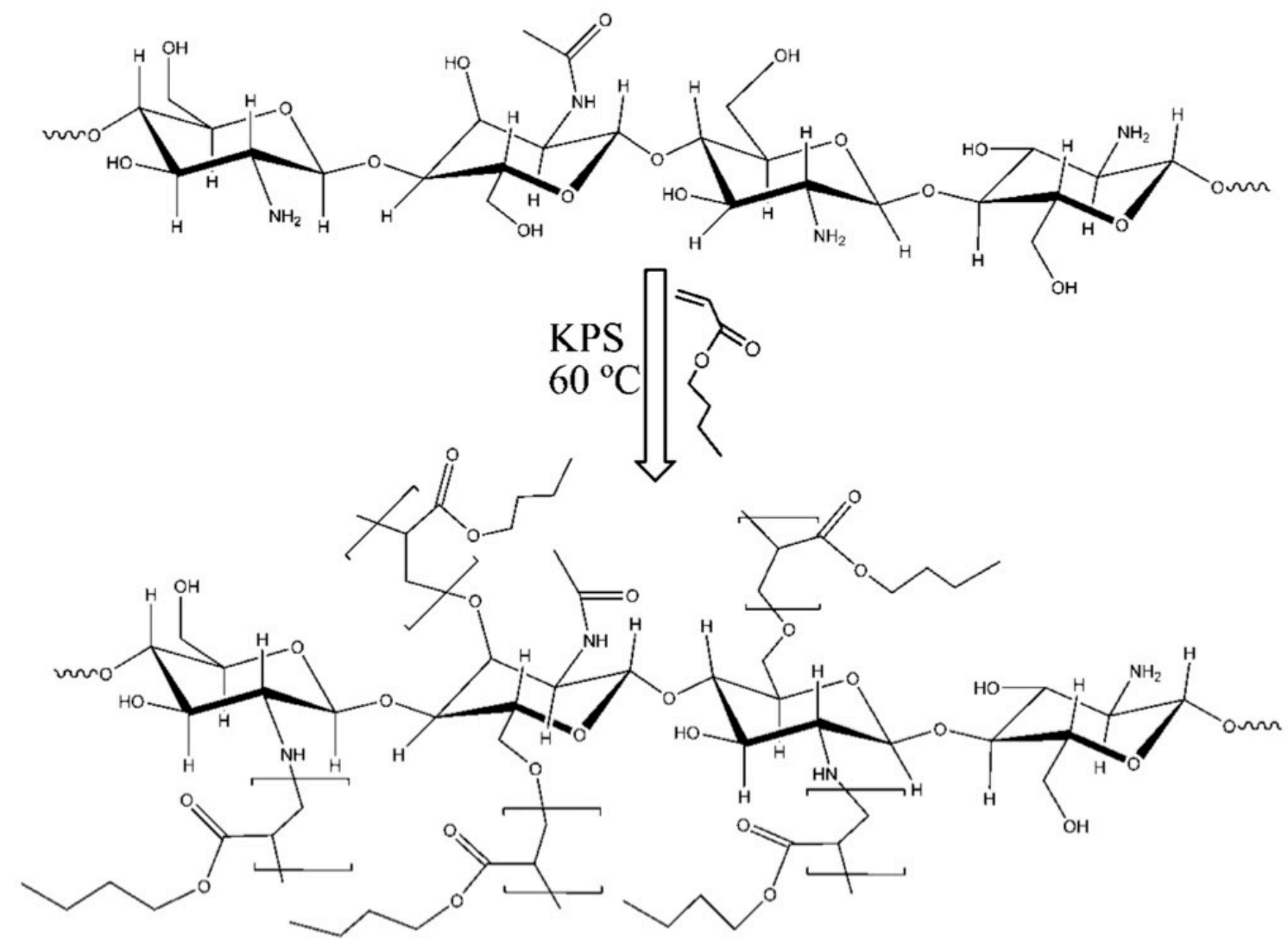

Fig. 1. Scheme of the reaction of the chitosan-g-butyl acrylate.

Table 2

Grafting percentage, reaction efficiency, particle sizes and polydispersity index.

\begin{tabular}{lllll}
\hline Sample & Grafting (\%) & Efficiency $(\%)$ & $\mathrm{d}_{\mathrm{z}}$-average diameter $(\mathrm{nm})$ & PdI \\
\hline QSA50 & 39.0 & 78.0 & 394.2 & 1.220 \\
QPA50 & 45.5 & 91.1 & 265.1 & 1.107 \\
QSA100 & 93.8 & 93.8 & 564.6 & 1.142 \\
QPA100 & 91.5 & 91.5 & 232.6 & 1.069 \\
\hline
\end{tabular}

Casted films $(\approx 100 \mu \mathrm{m})$ were easy to manipulate. In Fig. 1a scheme of the reaction is presented.

Grafting and efficiency results reported in Table 2 show that the synthesis method is satisfactory in order to get a natural-synthetic hybrid polymer with low content of acrylic homopolymer. Polymer grafting with radical initiators (KPS in this work) produces radicals in specific sites contributing to the grafting reaction. On the other hand, in the radical initiation made through photo-polymerization, the UV light directly generates free radicals on vinyl monomers and thereby an increasing homopolymerization (Harish Prashanth \& Tharanathan, 2003). The water soluble KPS dissolves in the chitosan aqueous solution and the radicals $\left(\cdot{ }^{\circ} \mathrm{SO}_{4}{ }^{-}\right)$abstract hydrogen of the amino groups (and probably of the $\mathrm{OH}$ groups) from the chitosan macromolecules, reacting with the $n$-butyl acrylate monomers and grafting to the macromolecules.

As claimed by other authors in similar systems (e.g., Ávila et al., 2012), the grafting efficiency increases with the increment of the acrylic monomer content in the QS (DD \% =78.2) grafted samples. In QP grafted samples there was not observed a significant improvement in the efficiency when the content of the acrylate monomer was increased. The better efficiency measured for the QP samples can be attributed to the content of amino groups in the chitosan structure; i.e. more grafting sites. With high concentration of acrylate monomer, this effect is negligible because of the increase of the rate of homopolymerization as a consequence of a higher probability to meet an acrylate monomer molecule.

Infrared spectroscopy is a very useful technique to evaluate the graft in polymers, due to it recognizes specific functional groups.

As can be seen in Fig. 2, IR spectra show characteristic absorption bands of chitosan at $3500-3000 \mathrm{~cm}^{-1}(\mathrm{O}-\mathrm{H}$ and $\mathrm{N}-\mathrm{H})$, $1644 \mathrm{~cm}^{-1}$ (Amide I), $1582 \mathrm{~cm}^{-1}$ ( $-\mathrm{NH}_{2}$ bending, Amide II) and $1376 \mathrm{~cm}^{-1}$ (- $\mathrm{CH}_{2}-$ bending). The absorption bands at $1151 \mathrm{~cm}^{-1}$ (anti-symmetric stretching of the $\mathrm{C}-\mathrm{O}-\mathrm{C}$ group), $1066 \mathrm{~cm}^{-1}$ and $1029 \mathrm{~cm}^{-1}$ (skeletal vibrations involving the $\mathrm{C}-\mathrm{O}$ stretching) are characteristic of saccharide structures. On the other hand, grafted samples show a strong carbonyl peak around $1730 \mathrm{~cm}^{-1}$ and $\mathrm{C}-\mathrm{H}$ stretching peaks at $2958 \mathrm{~cm}^{-1}$ indicating the presence of $n$-BuA chains.

Fig. 2 reveals the absence of the carbonyl band in pure chitosan and the presence, at $1728 \mathrm{~cm}^{-1}$, in pure pBuA. As mentioned above, grafted samples present their bands displaced with respect to the pure $\mathrm{pBuA}$ wavenumber indicating some interaction of $\mathrm{pBuA}$ with the chitosan matrix. Therefore, it could be concluded that the free hydroxyl groups of the chitosan chains could interact via hydrogen bonds with the pBuA carbonyl groups. When the acrylate percentage increases, the non-interacting carbonyl groups increase (i.e., more long chains and, therefore, less proportion of interacting groups); consequently, the band intensity has an increasing 


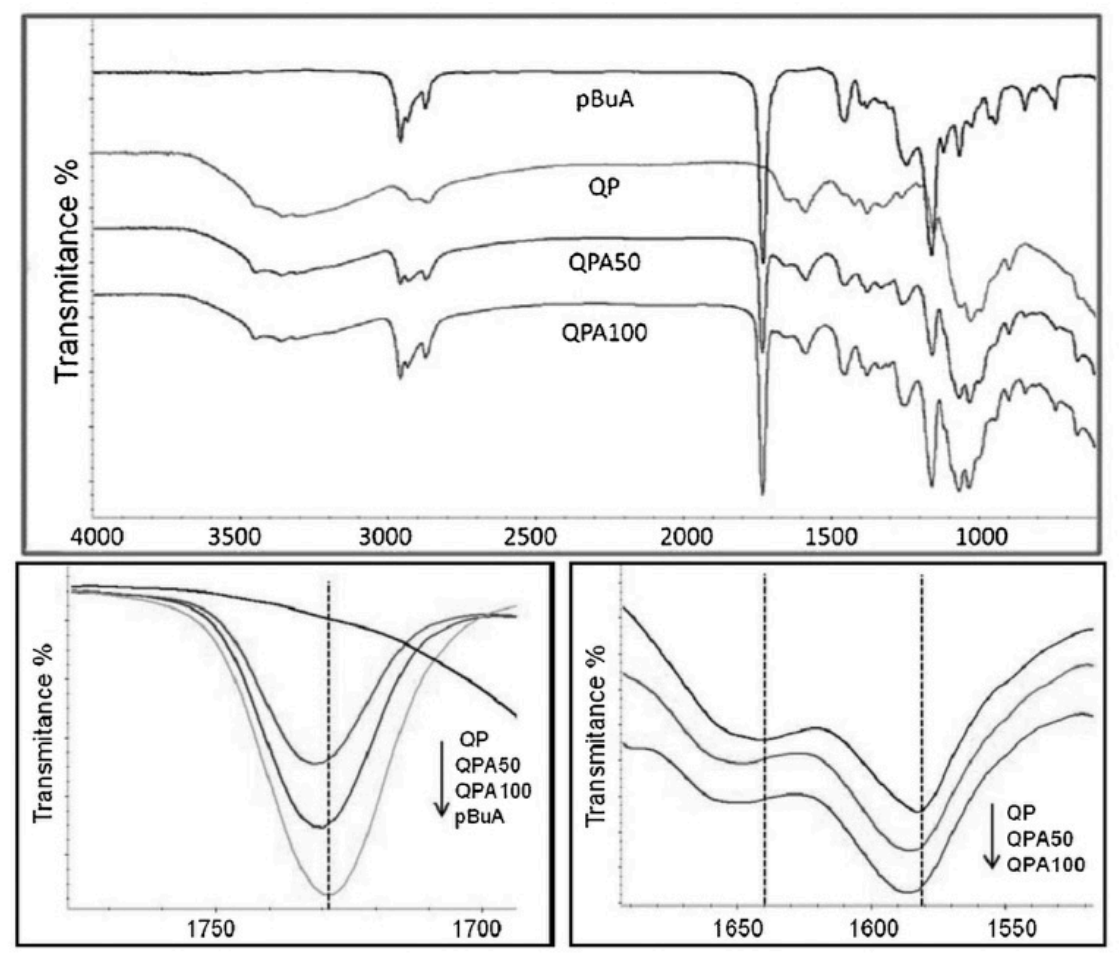

Fig. 2. Top: FT-IR spectra of poly(n-butyl acrylate), QP, QPA50 and QPA100 films. Bottom left: carbonyl-attributed band; bottom right: amino and amide groups-attributed bands. For clarity, the spectra were shifted vertically.

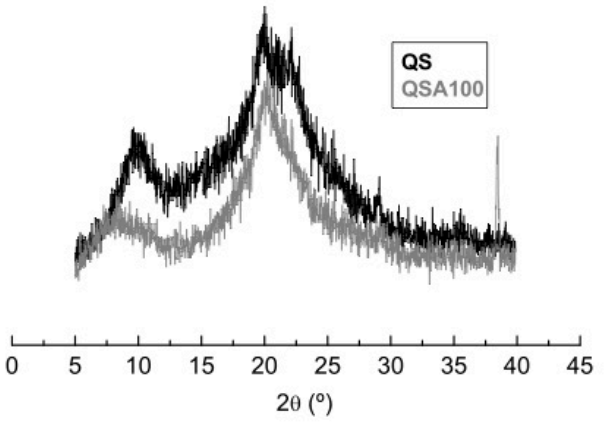

Fig. 3. XRD spectra obtained for QS and QSA100 samples.

contribution of these carbonyl groups and, as a result, a lower displacement in the wavenumber of the band is observed.

In Fig. 2, it can also be seen than both Amide I and Amide II bands of chitosan (1638 and $1582 \mathrm{~cm}^{-1}$, respectively) appears at lower wavenumbers in the pristine biopolymer than the revealed in the grafted samples. This behavior would confirm the grafting in the $\mathrm{NH}$ group; on the other hand, the decrease in the broad band centered at $3300 \mathrm{~cm}^{-1}$ (overlapped $\mathrm{OH}$ and $\mathrm{NH}$ stretching bands) could also be attributed to the grafting of $n$-BuA throughout the hydroxyl groups.

Changes in the chitosan structure after grafting were further investigated by means of powder XRD. In Fig. 3, the spectra obtained for a pure chitosan and a grafted sample are shown. For sake of clarity, only two spectra, one of a pure chitosan and the corresponding sample with the highest grafting percentage are presented. These two XRD spectra were chosen due to they present the bigger differences between each other.

QS chitosan XRD is characterized by two scattering angles at $2 \theta=10^{\circ}$ and $2 \theta=20^{\circ}$, named Form I and II, which are represented by an hydrated and an anhydrous crystals, respectively (Samuels, 1981). In the literature, the presence of an additional peak at $2 \theta$ about $22^{\circ}$ has been attributed to the allomorphic tendon form of chitosan (Chassary, Vincent and Guibal, 2004), which was found to produce a strong decrease in heavy metals sorption capacities (Jaworska, Sakurai, Gaudon and Guibal, 2003). The crystalline structure of chitosan is due to strong intermolecular and intramolecular hydrogen bonds between hydroxyl and amino groups.

On the other hand, for the QSA100 sample it can be observed a decrease in the intensity of the peak at $2 \theta=10^{\circ}$ and a shift of the maximum of this peak down to $8.3^{\circ}$. The lower intensity could be related to a crystallinity decrease due to the incorporation of $\mathrm{pBuA}$ chains along the biopolymer backbone, and/or with the decrease of the free $\mathrm{NH}_{2}$ and $\mathrm{OH}$ sites in which water could bond, since crystal Form I is related to a hydrated one (Ogawa, Yui and Miya, 1992). The shift in the peak position, i.e. the increase in the interlayer distance, can also be attributed to the grafting process. The disappearance of the signal at $2 \theta=22^{\circ}$ would indicate a loss of crystallinity and it could be of interest for heavy metal adsorption usages.

As can be seen in Fig. 4, TEM images reveal an spherical and well-defined core-shell morphology in the QSA50 dispersion with poly ( $n$-butyl acrylate) rich cores and chitosan shells; particle sizes around $200 \mathrm{~nm}$ were measured. Different content of the acrylic moiety could be observed inside the particles and pure chitosan particles are also present in the sample. In other grafted samples a similar morphology is observed.

Dynamic light scattering measurement, as shown in Table 2, indicates that highly monodispersed particles are obtained. Parti- 


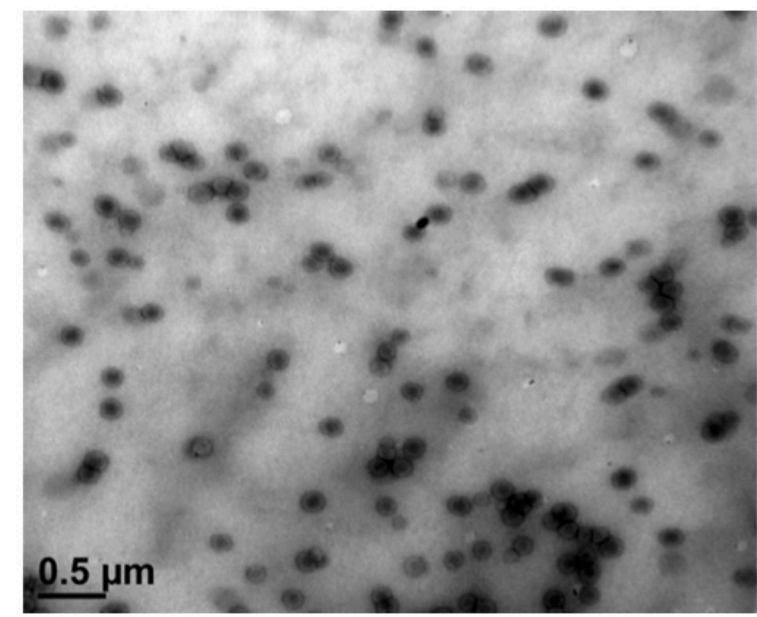

Fig. 4. TEM micrograph of $Q S A 50$ dispersion.

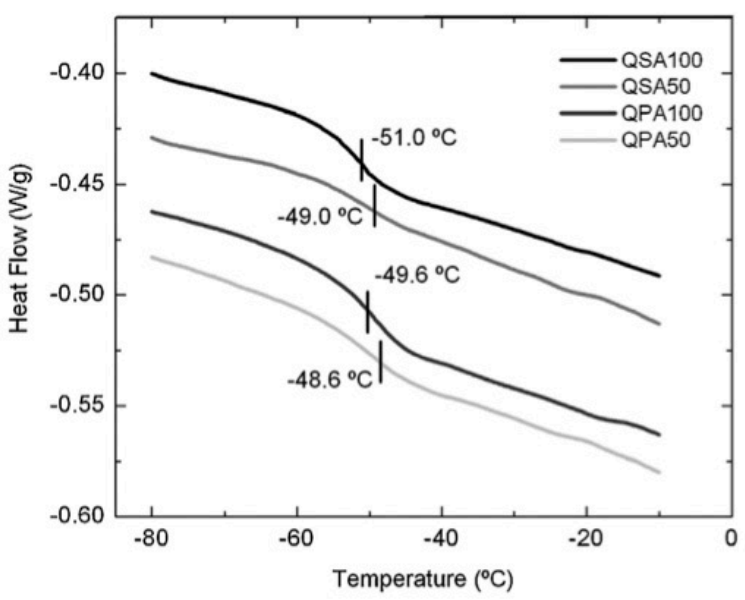

Fig. 5. DSC scans of the grafted samples labeled in the inset. Forclarity, thermograms were shifted vertically.

cle size depends on the DD\% of the pristine chitosan. In QP samples $(\mathrm{DD} \%=95.2)$, values of about $250 \mathrm{~nm}$ were found in both $1: 0.5$ and $1: 1$ chitosan:BuA ratio, with a very sharp distribution of sizes. On the other hand, QS samples (78.2 DD\%) shows sizes of 400 and $560 \mathrm{~nm}$ in the different chitosan:BuA ratios. Less grafting sites in QS samples involve a major growth of $\mathrm{pBuA}$ chains, giving bigger particles. Moreover, QS molecular weight also influence in grafted particle size.

The difference in sizes observed by TEM and by the Zetasizer instrument is related to the swollen state and the measurement of the hydrodynamic radius of the particles in the Zetasizer instrument.

As can be seen in Fig. 5 , DSC thermograms show $T_{\mathrm{g}}$ values of -51 and $-49^{\circ} \mathrm{C}$ for QSA100 and QSA50, respectively; and -49.6 and $-48.6^{\circ} \mathrm{C}$ for QPA100 and QPA50. Since for poly(n-butyl acrylate) a $T_{\mathrm{g}}$ value of $-54^{\circ} \mathrm{C}$ was reported (Jakubowski et al., 2008; Zhang et al., 2013), it is reasonable to assume that the pBuA chains have a reduced mobility due to the grafting process. As can be observed, both grafting ratio and $\mathrm{DD} \%$ of the chitosan influence the $T_{\mathrm{g}}$ values of the grafted samples. The increase of the grafting ratio generates longer pBuA chains and, as discussed above, when analyzing the FT-IR spectra, a lower interaction with the chitosan backbone with the consequent increase of the mobility of the chains. In the same way, when more grafting sites (i.e., a higher DD\%) in which the $\mathrm{BuA}$ monomer could attach and polymerize are available, a lower growth of every $\mathrm{pBuA}$ chains is reached generating an opposite effect to that described in the previous sentence. Besides, the absence of a $T_{\mathrm{g}}$ around $-54^{\circ} \mathrm{C}$ confirm that homopolymer chains were completely removed.

As can be seen in Fig. 6.a the water swelling degree (WS\%) is affected by the percentage of acrylic component and $\mathrm{DD} \%$ of the pure chitosan. In fact, for the QS grafted samples (DD\%=78.2) a gradual decrease in the WS\% values is observed, meanwhile in the QP grafted samples the decrease of this parameter is abrupt reaching a $25 \%$ of WS\% in the QPA 100 sample, equivalent at a diminution of about $75 \%$.

Several authors(Tager, 1972; Taghizadeh \& Davari, 2006: Baskar \& Sampath Kumar, 2009) concluded that DD\% is one of the factors, but not necessarily the most important, that affect the water swelling in chitosan. Tager (1972) claimed that the other factors are: nature of polymer and solvent, flexibility of the polymer chains, molecular mass, crystal structure and chemical crosslinks. Moreover, Taghizadeh and Davari (2006) concluded that, in chitosan samples with similar DD\% to those used in the present work, the main factors involved in the swelling are the crystallinity and the molecular weight. It is known that the hydrophilic nature of chitosan comes from the presence of free amino groups in the $\mathrm{C}-2$ and hydroxyl groups in the on C-3 and C-6 ones. During grafting process, a significant number of free amino groups and hydroxyl groups are blocked by the growing chains of the pBuA. In our case, the role of the $\mathrm{N}$-acetylated groups is still important due to both the number of graft sites and the possibility to found $\mathrm{N}$-acetylglucosamine blocks (Rinaudo, 2006). For instance, in the QS (DD\% = 78.2) grafted samples, the acrylic monomer graft into less sites along the chitosan molecule; moreover, if the $\mathrm{N}$-acetylated units form blocks, the hydrophobicity given by the acrylic moiety would be restricted to zones in the chitosan chain.

Baskar and Sampath Kumar (2009) concluded that DD\% in chitosan does not affect the water contact angle. As can be seen in Fig. 6b there are non significant differences, within the error bars, between the water contact angle measured for the QS and QP chitosan samples. However, for the QP samples values of this parameter are always systematically higher than the QS ones, that means that there are more hydrophobic surface in the QP samples. As can be seen in the figure, there are not differences, between the experimental scatter, among the values of the mentioned parameter obtained for pure and grafted samples. This behavior could be related to the core-shell morphology of the dispersion particles and the film formation process in which the solvent (i.e., water) evaporation front moves the chitosan moiety to the film surface.

From the industrial point of view, it is very attractive the possibility to found in the grafted samples both chitosan in the surface and a lower water swelling. This could be beneficial for several applications due to the biocompatible and antimicrobial properties of the chitosan-based films with an enhancement of the performance against water.

Free volume plays an important role in different film properties like permeability and mechanical response. To further analyze the free nanohole volume and the nanostructural variations in the chitosan and grafted samples, PALS measurements were carried out. As a result, $\tau_{0-\text { Ps }}$ and $I_{0-\text { Ps }}$ values were obtained for each sample studied. In Table 3 the obtained results are shown. o-Ps lifetimes measured for pure chitosan samples are in good agreement with those reported by Sharma et al. (2013) and Chaudhary, Went, Nakagawa, Buckman, and Sullivan (2010).

As can be seen in Table 3 , in grafted samples $\tau_{0 \text {-Ps }}$ is higher than that measured in pure chitosan and that this lifetime increases for higher grafting ratios. These results are discussed below. 

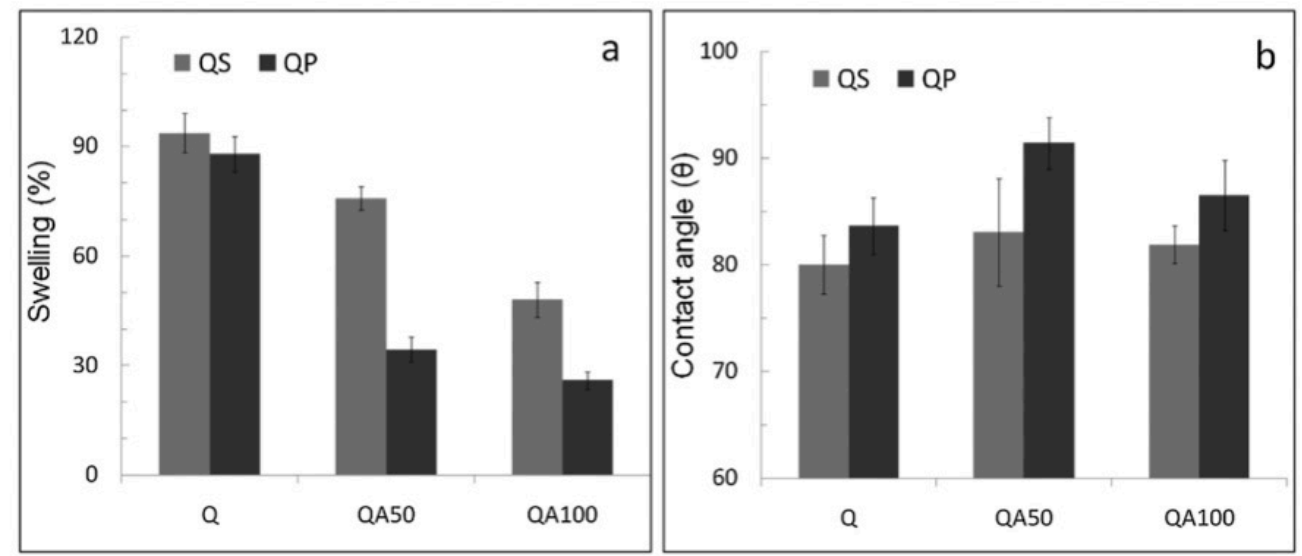

Fig. 6. Values of the swelling degree (6a) and contact angle (6b) measured in pure chitosan and grafted samples.

Table 3

ortho-Positronium lifetimes and their associated intensities obtained measuring pure chitosans and grafted samples.

\begin{tabular}{lll}
\hline Sample & $\tau_{\text {o-Ps }}(\mathrm{ns})$ & $I_{0-\mathrm{Ps}_{\mathrm{s}}(\%)}$ \\
\hline QS & $1.77 \pm 0.02$ & $16 \pm 0.3$ \\
QP & $1.70 \pm 0.02$ & $14 \pm 0.3$ \\
QSA50 & $2.17 \pm 0.04$ & $13 \pm 0.5$ \\
QPA50 & $2.35 \pm 0.03$ & $13 \pm 0.3$ \\
QSA100 & $2.45 \pm 0.04$ & $14 \pm 0.4$ \\
QPA100 & $2.50 \pm 0.03$ & $15 \pm 0.4$ \\
\hline
\end{tabular}

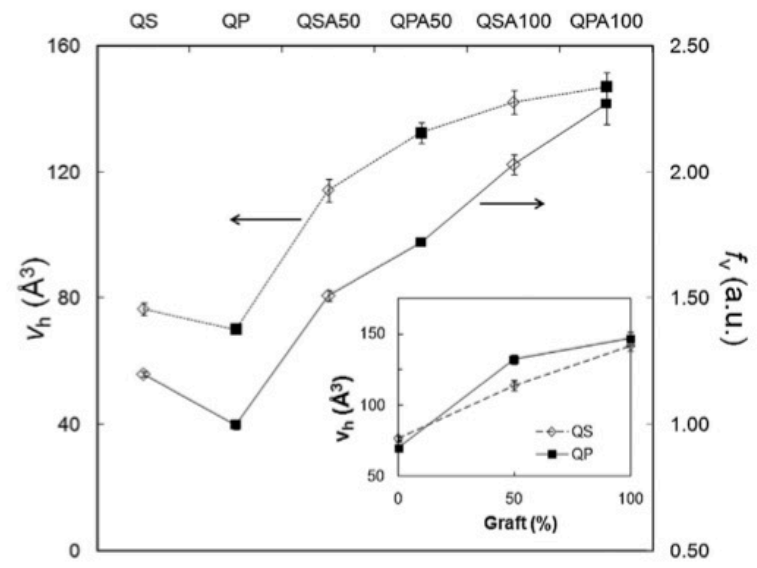

Fig. 7. Free nanohole volumes and fractional free volumes obtained for pure and grafted samples. Inset: $v_{\mathrm{h}}$ values respect to graft percentage.

Using Eq. (5) and (6), both the free nanohole volume and the fractional free volume values were obtained for each sample, results were plotted in Fig. 7. As can be seen, the $v_{\mathrm{h}}$ value for the QS sample is about $10 \%$ higher than that corresponding to the QP sample. In the case of the grafted chitosans, a noticeable increment in the $v_{\mathrm{h}}$ values when compared to those of pure chitosan samples is observed.

Furthermore, in the inset of Fig. 7, $v_{\mathrm{h}}$ values for the different samples studied were represented as a function of the graft percentage. In the case of the samples of the QS series, a linear behavior is observed; meanwhile for the QP series the initial $v_{\mathrm{h}}$ value obtained for QP present an important increment when the graft percentage is $50 \%$; for the highest graft percentage, $v_{\mathrm{h}}$ value of QPA100 sample coincide with the obtained for the QSA100.
In the case of the fractional free volume, the value of this parameter for the QS sample is $20 \%$ higher than the obtained for QP one. The $f_{v}$ values for both QS and QP series linearly increase with the graft percentage. In $\mathrm{QS}$ series the increment in $f_{\mathrm{v}}$ value between the pure chitosan and the QSA100 sample is 70\%, while in QP series this increment is near $130 \%$

The differences observed in the $v_{\mathrm{h}}$ and $f_{\mathrm{v}}$ values for QS and QP pure chitosan samples can be related to differences in their respective molecular weights and deacetylation degree. It must be taken into account that more voluminous groups are present in the acetylated moiety. On the other hand, the different evolutions of $v_{\mathrm{h}}$ with the graft percentage for both kinds of chitosan samples can be interpreted in the same terms of those argued for the analysis of the swelling results; i.e. the main parameter responsible of the behaviors observed, is the number of grafting sites (related with $\mathrm{DD} \%$ ) existing in the QP chitosan samples.

Furthermore, the increment of the o-Ps lifetime, and therefore of $v_{\mathrm{h}}$, for the grafted samples with respect to the pure chitosan ones could be attributed to the rupture of specific interactions (such as the $\mathrm{H}$ bonds) among polar groups in the biopolymer due to the graft of $\mathrm{pBuA}$ in the functional groups $\left(\mathrm{NH}_{2}, \mathrm{OH}\right)$; this procedure implies the separation of the chitosan chains breaking the typical rigid rod-structure of the polysaccharide.

Lin et al. (2010) found that a chitosan coat on starch-based films produced lower water swelling and diffusion coefficient values than the uncoated samples, the authors related this behavior with a reduction of the free nanohole volumes. Conversely, as seen in Fig. 6a grafted samples have a noticeable reduction of the swelling degree with respect to that of the pure chitosan, even when $v_{\mathrm{h}}$ values corresponding to the grafted samples are much bigger. This behavior could be associated with the hydrophobic nature of the acrylic component used to prepare our grafted samples. On the other hand, Lizama, López-Castañares, Vilchis, and Vázquez (2003) used PALS to obtain the free nanohole volume of a poly(butyl acrylate) homopolymer sample. The $v_{\mathrm{h}}$ value reported by these authors is in very good agreement with that obtained for the QA100 sample. Under this scenario, the main contribution to the long-lived lifetime to o-Ps measured in the grafted samples could be attributed to Ps annihilations in rich-acrylate regions.

Even when the chitosan/poly( $n$-butylacrylate) samples analyzed in the present work are chemically bonded and that they are not completely immiscible (see IR-FT results), a possible contribution to o-Ps annihilations coming from the interphase zones cannot be discarded as reported Liu and Jean (1995) and Mansilla, Silva, Salgueiro, Marzocca, and Somoza (2012). 


\section{Conclusions}

In the present work a study on the synthesis and characterization of natural/synthetic hybrid polymers is presented. From, the results obtained, it can be concluded that:

On the synthesis:

- $n$-Butyl acrylate was successfully grafted onto a chitosan backbone in an aqueous surfactant-free reaction medium, obtaining stable dispersions and freestanding films once casted.

- The grafting process was confirmed by IR spectroscopy analysis.

- High grafting efficiency percentage was obtained. This parameter depends on the DD\% of the chitosan.

On the characterization:

- Interaction between biopolymer and grafted acrylic was revealed by FTIR spectroscopy.

- Well defined core-shell morphology was obtained in grafted samples.

- Film water swelling is reduced by the grafting, without decreasing hydrophilicity.

- Free nanohole volumes and their fractions were increased by grafting; while $f_{\mathrm{v}}$ follows a lineal increment with respect to grafting ratio, $v_{\mathrm{h}}$ behavior depends on the pristine chitosan.

\section{Acknowledgments}

We acknowledge for funding Agencia Nacional de Promoción Científica y Tecnológica (Argentina) (PICT 2011-0238 and PICT 2011-1088), Consejo Nacional de Investigaciones Científicas y Técnicas (Argentina)(PIP\#112-201101-00793), Comisión de Investigaciones Científicas de la Provincia de Buenos Aires and SECAT (UNCPBA). Authors thank F. Irassar for his useful support in XRD experiments. PSA and CM are members of CONICET, AS and JIA are members of CICPBA.

\section{References}

Armarego, W. L. F., \& Chai, C. (2013). Purification of laboratory chemicals (7th Ed.) Elsevier Inc., pp. 123

Averous, L., Moro, L., Dole, P., \& Fringant, C. (2000). Properties of thermoplastic blends: starch-polycaprolactone. Polymer, 41(11), 4157-4167,

Ávila, A., Bierbrauer, K., Pucci, G., López-González, M., \& Strumia, M. (2012). Study of optimization of the synthesis and properties of biocomposite films based on grafted chitosan. Journal of Food Engineering, 109,752-761.

Baskar, D., \& Sampath Kumar, T. S. (2009). Effect of deacetylation time on the preparation: properties and swelling behavior of chitosan films. Carbohydrate Polymers, 78, 767-772

Baxter, A., Dillon, M., Taylor, K., \& Roberts, G. (1992). Improved method for i.r determination of the degree of $\mathrm{N}$-acetylation of chitosan. International Joumal of Biological Macromolecules, 14, 166-169.

Bravo-Osuna, I., Schmitz, T., Bernkop-SchnÛrch, A., Vauthier, C., \& Ponchel, G. (2006). Elaboration and characterization of thiolated chitosan-coated acrylic nanoparticles. International Journal of Phamaceutics, 316, 170-175.

Chassary, P.. Vincent, T., \& Guibal, E. (2004). Metal anion sorption on chitos an and derivative materials: a strategy for polymer modification and optimum use. Reactive \& Functional Polymers, 60, 137-149.

Chaudhary, D., Went, M. R., Nakagawa, K., Buckman, S. J., \& Sullivan, J. P. (2010). Molecular pore size characterization with in chitosan biopolymer using positron annihilation lifetime spectroscopy. Materials Letters, 64, 2635-2637.

Chellapandian, M., \& Krishnan, M. R. V. (1998). Chitosan-poly (glycidyl methacrylate) copolymer for immobilization of urease. Process Biochemistry, 33(6), 595-600

Chen, Y., \& Tan, H. (2006). Crosslinked carboxymethyl chitosan-g-poly(acrylic acid) copolymer as a novel superabsorbent polymer. Carbohydrate Research, 341 . 887-896.

Chivrac, F., Pollet, E., \& Avérous, L. (2009). Progress in nano-biocomposites based on polysaccharides and nanoclays. Materials Science and Engineering R, 67, 1-17.

Consolati, G., Levi, M., \& Turri, S. (2001). Positron anni hilation and phase separation phenomena in polyure thane crosslinked coatings with different polyether phenomena in polyurethane
segments. Polymer, 42, 9723.

Costa-Júnior, E. S., Barbosa-Stancioli, E. F., Mansur, A. A. P., Vasconcelos, W. L. \& Mansur, H. S. (2009). Preparation and characterization of chitosan/poly(vinyl alcohol) chemically crosslinked blends for biomedical applications Carbohydrate Polymers, 76, 472-48

Eldrup, M., Lightbody, D., \& Sherwook, N. J. (1981). The temperature dependence of positron lifetimes in solid pivalic acid. Chemical Physics, 63, 51-58.

Ge, H., \& Wang, S. (2014). Thermal preparation of chitosan-acrylic acid superabsorbent: optimization, characteristic and water absorbency. Carbohydrate Polymers, 113, 296-303.

Giebel, D., \& Kansy, J. (2011). A new version of LT program for positron lifetime spectra analysis. Materials Science Forum, 666, 138-141.

Harish Prashanth, K. V., \& Tharanathan, R. N. (2003). Studies on graft copolymerization of chitosan with synthetic monomers. Carbohydrate Polymers, 54, 343-351.

Ifuku, S., Iwasaki, M., Morimoto, M., \& Saimoto, H. (2011). Graft polymerization of acrylic acid onto chitin nanofiber to improve dispersibility in basic water. Carbohydrate Polymers, 90(1), 623-627.

Jakubowski, W., Juhari, A., Best, A., Koynov, K., Pakula, T., \& Matyjaszewski, K. (2008). Comparison of thermomechanical properties of statistical: gradient and block copolymers of isobornyl acrylate and $n$-butyl acrylate with various acrylate homopolymers. Polymer, 49, 1567-1578.

Jaworska, M., Sakurai, K., Gaudon, P., \& Guibal, E. (2003). Influence of chitosan characteristics on polymer properties: I: crystallographic properties. Polymer International, 52, 198-205.

Jean, Y. C. (1990). Positron annihilation spectroscopy for chemical analysis: a novel probe for microstructural analysis of polymers. Microchemical Journal, 42, 72

Jiang, X., Chen, L., \& Zhong, W. (2003). A new linear potentiometric titration method for the determination of deacetylation degree of chitosan. Carbohydrate Polymers, 54, 457-463.

Kirwan, M. J., \& Strawbridge,J. W. (2003). Plastics in food packaging. Food

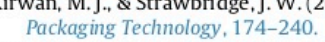

Kobayashi, Y., Zheng, W., Meyar, E. F., McGervey, J. D., Jamieson, A. M., \& Simha, R. (1989). Free volume and physical aging of poly(vinyl acetate) studied by positron annihilation. Macromolecules, 22, 2302

Li, Y., Liu, L., Shen, X., \& Fang, Y. (2005). Preparation of chitosan/poly(butyl acrylate) hybrid materials by radiation-induced graft copolymerization based on phthaloyl chitosan. Radiation Physics and Chemistry, 74, 297-301.

Lin, B., Du, Y., Li, Y., Liang, X., Wang, X., Deng, W.., \& Kennedy, J. (2010). The effect of moist heat treatment on the characteristic of starch-based composite materials coating with chitosan. Carbohydrate Polymers, 81, 554-559,

Liu, J., \& Jean, Y.C. (1995). Free-vol ume hole properties of polymer blends probed by positron annihilation spectroscopy: miscibility. Macromolecules, 28 ,

Lizama, B., López-Castañares, R., Vilchis, V., \& Vázquez, F. (2001). Morphological characterization of composite latex particles by positron annihilation lifetime spectroscopy. Materials Research Innovations, 5, 63-66.

Lloyd, L. L., Kennedy, J. F., Methacanon, P., Paterson, M., \& Knill, C. J. (1998) Carbohydrate polymers as wound management aids. Carbohydrate Polymers, $37,315-322$

Mansilla, M. A., Silva, L., Salgueiro, W., Marzocca, A. J., \& Somoza, A. (2012). A study about the structure of vulcanized natural rubber/styrene butadiene rubber blends and the glass transition behavior. Journal of Applied Polymer Science, $125,992-999$.

Masri, M. S., \& Friedman, M. (1972). Mercury uptake by polyamine-carbohydrates. Environmental Science and Technology, 6(8), 745-746.

Muzzarelli, R. A., Isolati, A., \& Ferrero, A. (1974). Chitosan membranes. Ion Exchange and Membranes, 1(4), 193-196.

Nakanishi, H., Wang, S. J., \& Jean, Y. C. (1988). Microscopic surface tension studied by positron annihilation. In S. C. Sharma (Ed.), Positron annihilation studies of by positron annihilation. In S. C. Sharma (Ed.), Post
fluids (pp. 292-298). Singapore: World Sci. Pub.

Ogawa, K., Yui, T., \& Miya, M. (1992). Dependence on the preparation procedure of the polymorphism and crystallinity of chitosan membranes. Bioscience, Biotechnology, and Biochemistry, 56(6), 858-862

Özbaș, Z., \& Gürdağ, G. (2015). Swelling kinetics, mechanical properties, and release characteristics of chitosan-based semi-IPN hydrogels. Journal of Applied Polymer Science, 132(16), 41886.

Rhim, J.-W., \& Ng, P. K. W. (2007). Natural biopolymer-based nanocomposite films for packaging ap plications. Critical Reviews in Food Science and Nutrition, 47, 411-433.

Rinaudo, M. (2006). Chitin and chitosan: properties and ap plications. Progress in Polymer Science, 31,603-632.

Rinaudo, M. (2012). Physical properties of chitosan and derivatives in sol and gel states. In B. Sarmento, \& J. das Neves (Eds.), Chitosan-based systems for biopharmaceuticals: delivery, targeting, and polymer therapeutics. John Wiley \& Sons, Ltd.

Samuels, R. J. (1981). Solid state characterization of the structure of chitosan films. Journal of Polymer Science: Polymer Physics Edition, 19, 1081-1105.

Shankar, P., Gomathi, T., Vijayalakshmi, K., \& Sudha, P. N. (2014). Comparative studies on the removal of heavy metals ions onto cross linked chitosan-g-acrylonitrile copolymer. International Journal of Biological Macromolecules, 67, 180-188.

Sharma, S. K., Bahadur, J., Patil, P. N., Mahes hwari, P., Mukherjee, S., Sudarshan, K. \& Pujari, P. K. (2013). Revealing the nano-level molecular packing in chitosan-NiO nanocomposite by using positron annihilation spectroscopy and small-angle X-ray scattering. ChemPhysChem, 14, 1055-1062.

Singh, D. K., \& Ray, A. R. (1998). Characterization of grafted chitosan films. Carbohydrate Polymers, 36(2-3), 251-255. 
Singh, V., Sharma, A. K., Tripathi, D. N., \& Sanghi, R. (2009).

Poly(methylmethacrylate) grafted chitosan: an efficient ads orbent for anionic azo dyes. Joumal of Hazardous Materials, 161(2-3), 955-966.

Tager, A. (1972). Physical chemistry of polymers. pp. 331-334. Moscow: Mir Publishers.

Taghizadeh, S. M., \& Davari, G. (2006). Preparation, characterization: and swelling behavior of $\mathrm{N}$-acetylated and deacetylated chitosans, Carbohydrate Polymers, 64, 9-15.

Tao, S. J. (1972). Positronium annihilation in molecular substances. Journal of Chemical Physics, 56, 5499-5510,

van den Broek, L. A. M., Knoop, R. J. I., Kappen, F. H. J., \& Boeriu, C. G. (2015),

Chitosan films and blends for packaging material. Carbohydrate Polymers, 116 $237-242$.
Vartiainen, J., Tammelin, T., Pere, J., Tapper, U., \& Harlin, A. (2010). Biohybrid barrier films from fluidized pectin and nanoclay. Carbohydrate Polymers, 82(3), 989-996

Wang, Y. Y., Nakanishi, H., Jean, Y. C., \& Sandreczki, T. C. (1990). Positron annihilation in amine-cured epoxy polymersdpressure dependence. Journal of Polymer Science Part B: Polymer Physics, 28, 1431.

Xie, F., Pollet, E., Halley, P. J., \& Avérous, L. (2013). Starch-based

nano-biocomposites. Progress in Polymer Science, 38, 1590-1628.

Zhang, L., Cao, Y., Wang, S., Czech, Z., Shao, L., \& Bai, Y.(2013). Synthes is of poly

(n-butyl acrylates) by a novel microemulsion polymerization for PSAs applications. International Journal of Adhesion \& Adhesives, 47, 69-72. 